\title{
Some Rigid-Body Constraint Varieties Generated by Linkages
}

J.M. Selig

\begin{abstract}
The set of rigid-body displacements allowed by three simple open-chain linkages are studied. These linkages consist of a cylindrical and spherical joint: the CS dyad, a revolute, a prismatic and a spherical joint: the RPS linkage, two revolutes and a spherical joint: the RRS linkage. Using the Study quadric to represent the group of all rigid-body displacements the constraint varieties for these examples are found. In the case of the CS and RPS linkages these are found to be quartic hypersurfaces while the constraint variety for the RRS linkage is a hypersurface of degree 8 . Finally it is shown that all three constraint varieties are linear projections of a Segre variety in $\mathbb{P}^{15}$.
\end{abstract}

Key words: Rigid-body displacements, open-chain linkages, constraint varieties.

\section{Introduction}

Previous work $[6,7]$ studied the set of all proper rigid-body displacements which transformed a point in such a way that it remained on a fixed plane or a fixed sphere. These sets of rigid displacements are important in kinematics because they can also be viewed as the possible displacements achievable by an ES or an SS dyad respectively. It was shown that if the space of rigid displacements is represented by the Study quadric, then the constraint manifolds for the point-plane and point-sphere constraints are the intersection of the Study quadric with another quadric hypersurface in $\mathbb{P}^{7}$.

The present work follows some work of McCarthy and co-workers [4] who investigated the set of displacements achievable by certain open-chain linkages. Some of this work is repeated here using the Study quadric as a model for the group of

J.M. Selig

London South Bank University, London SE1 0AA, U.K.

e-mail: seligjm@1sbu.ac.uk 


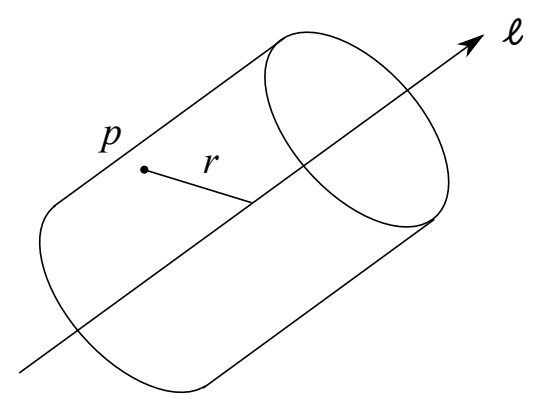

Fig. 1 A Point on a Cylinder.

all rigid-body displacements. In a final section some general remarks are made concerning the geometry of the constraint varieties produced by the three examples considered below.

It is convenient to use the Clifford algebra $\mathrm{Cl}(0,3,1)$ to perform some of the geometric computations. This algebra is particularly well suited to Euclidean geometry and contains the dual quaterions as a subalgebra. Unfortunately limitations on space preclude a review of this material, however a complete introduction to this algebra and its use for geometric computation may be found in [5, Chap. 10].

\section{The CS Dyad}

The first linkage considered is a chain consisting of a cylindrical joint together with a spherical joint. Note that the same set of displacements could be achieved with a revolute joint, a prismatic joint and a spherical joint so long as the axes of the revolute and prismatic joints are parallel.

Another way to look at this set of possible displacements is as the set of group elements that preserve the incidence of a point with a cylinder. The point is the centre of the spherical joint and the axis of the cylinder is the axis of the cylindrical joint, see Fig. 1.

Using the Clifford algebra $C l(0,3,1)$, it is now easy to find the equation satisfied by the group elements which preserve this incidence. In this algebra a point is given by $p=p_{0} e_{1} e_{2} e_{3}+p_{1} e_{2} e_{3} e+p_{2} e_{3} e_{1} e+p_{3} e_{1} e_{2} e$ where the $p_{i}$ are the projective coordinates of the point and The $e_{i} \mathrm{~s}$ are the basis elements of the algebra. A general line has the form $\ell=u_{01} e_{2} e_{3}+u_{02} e_{3} e_{1}+u_{03} e_{1} e_{2}+u_{23} e_{1} e+u_{31} e_{2} e+u_{12} e_{3} e$, where the $u_{i j}$ are the Plücker coordinates of the line. In general the square of the distance from a point $p$ to a line $\ell$ is given by,

$$
r^{2}=(p \vee \ell)(p \vee \ell)^{-} / \ell \ell^{-}
$$

Here $\vee$ is the shuffle product, a derived product in the Clifford algebra and the superscript ()$^{-}$, denotes the Clifford conjugate of an element of the algebra. If the 
cylinder has axis $\ell$ and radius $r$ then the required rigid displacements $g$, will satisfy,

$$
\left(g p g^{-} \vee \ell\right)\left(g p g^{-} \vee \ell\right)^{-}=r^{2}\left(\ell \ell^{-}\right) .
$$

If the point $p$ lies on the cylinder to begin with, then this can be written,

$$
\left(g p g^{-} \vee \ell\right)\left(g p g^{-} \vee \ell\right)^{-}-(p \vee \ell)(p \vee \ell)^{-}\left(g g^{-}\right)^{2}=0 .
$$

For a fixed cylinder this equation is a homogeneous quartic in $\mathbb{P}^{7}$.

To make this a little clearer and to investigate some of the properties of these constraint varieties a particular example is introduced. Assume that the point $p$ is initially located at the origin and the line $\ell$ is parallel to the $z$-axis but displaced a distance $r$ in the $x$-direction. In the Clifford algebra these elements are given by

$$
p=e_{1} e_{2} e_{3} \quad \text { and } \quad \ell=e_{1} e_{2}-r e_{2} e .
$$

A general group element in this algebra has the form,

$$
g=a_{0}+a_{1} e_{2} e_{3}+a_{2} e_{3} e_{1}+a_{3} e_{1} e_{2}+c_{0} e_{1} e_{2} e_{3}+c_{1} e_{1} e+c_{2} e_{2} e+c_{3} e_{3} e,
$$

where the coefficients $a_{i}$ and $c_{i}$ satisfy $a_{0} c_{0}+a_{1} c_{1}+a_{2} c_{2}+a_{3} c_{3}=0$, the quadratic equation defining the Study quadric. Using this to transform the point gives,

$$
\begin{aligned}
g p g^{-}= & \left(a_{0}^{2}+a_{1}^{2}+a_{2}^{2}+a_{3}^{2}\right) e_{1} e_{2} e_{3}+2\left(a_{0} c_{1}-a_{1} c_{0}+a_{2} c_{3}-a_{3} c_{2}\right) e_{2} e_{3} e+ \\
& 2\left(a_{0} c_{2}-a_{1} c_{3}-a_{2} c_{0}+a_{3} c_{1}\right) e_{3} e_{1} e+2\left(a_{0} c_{3}+a_{1} c_{2}-a_{2} c_{1}-a_{3} c_{0}\right) e_{1} e_{2} e
\end{aligned}
$$

and hence,

$$
\begin{aligned}
g p g^{-} \vee \ell=- & 2 r\left(a_{0} c_{2}-a_{1} c_{3}-a_{2} c_{0}+a_{3} c_{1}\right) e- \\
& 2\left(a_{0} c_{2}-a_{1} c_{3}-a_{2} c_{0}+a_{3} c_{1}\right) e_{1}+ \\
& \left(2\left(a_{0} c_{1}-a_{1} c_{0}+a_{2} c_{3}-a_{3} c_{2}\right)-r\left(a_{0}^{2}+a_{1}^{2}+a_{2}^{2}+a_{3}^{2}\right)\right) e_{2} .
\end{aligned}
$$

Finally the quartic equation sought is,

$$
\begin{aligned}
& \left(g p g^{-} \vee \ell\right)\left(g p g^{-} \vee \ell\right)^{-}-(p \vee \ell)(p \vee \ell)^{-}\left(g g^{-}\right)^{2}= \\
& 4\left(a_{0} c_{1}-a_{1} c_{0}+a_{2} c_{3}-a_{3} c_{2}\right)^{2}+4\left(a_{0} c_{2}-a_{1} c_{3}-a_{2} c_{0}-a_{3} c_{1}\right)^{2}- \\
& 4 r\left(a_{0}^{2}+a_{1}^{2}+a_{2}^{2}+a_{3}^{2}\right)\left(a_{0} c_{1}-a_{1} c_{0}+a_{2} c_{3}-a_{3} c_{2}\right)=0 .
\end{aligned}
$$

Let us write,

$$
\begin{gathered}
F=\left(a_{0} c_{1}-a_{1} c_{0}+a_{2} c_{3}-a_{3} c_{2}\right)^{2}+\left(a_{0} c_{2}-a_{1} c_{3}-a_{2} c_{0}-a_{3} c_{1}\right)^{2}- \\
r\left(a_{0}^{2}+a_{1}^{2}+a_{2}^{2}+a_{3}^{2}\right)\left(a_{0} c_{1}-a_{1} c_{0}+a_{2} c_{3}-a_{3} c_{2}\right),
\end{gathered}
$$

so that the quartic constraint equation is $F=0$. Clearly $F$ vanishes when $a_{0}=$ $a_{1}=a_{2}=a_{3}=0$. This 3-plane also lies in the Study quadric $a_{0} c_{0}+a_{1} c_{0}+a_{2} c_{2}+$ $a_{3} c_{3}=0$ but does not correspond to any rigid-body displacement. This 3-plane of 


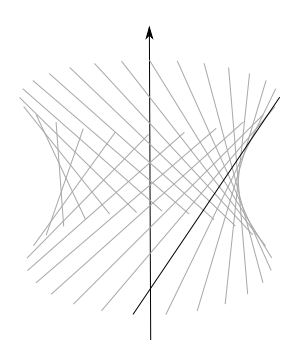

(a)

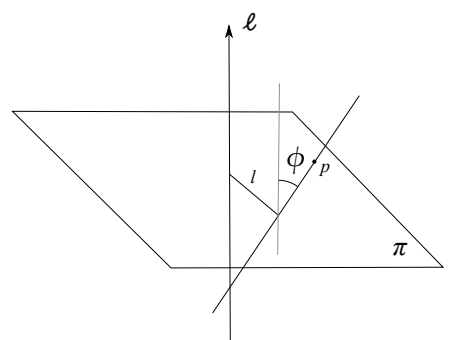

(b)

Fig. 2 A Point on a Hyperboloid.

ideal elements will be denoted $A_{\infty}$. The above shows that $A_{\infty}$ lies in this constraint variety. Moreover, it is easy to see that the partial derivatives $\partial F / \partial a_{i}$ and $\partial F / \partial c_{i}$ ( $i=0,1,2,3$ ) all vanish on $A_{\infty}$. So the 3 -plane is in fact singular in the quartic.

Notice that this result doesn't depend on the value of $r$, the radius of the cylinder. Moreover, the corresponding equation for a CS linkage with a different axis can be found by an action of the group of rigid-body displacements. However, the 3-plane $A_{\infty}$ is invariant under the action of the group and hence this 3-plane will be singular in the constraint variety for any CS linkage.

\section{The RPS Linkage}

Next we look at the RPS linkage. The group elements generated by such a linkage can be considered as the group elements that constrain the centre of the spherical joint to remain on a cylindrical hyperboloid. This cylindrical hyperboloid is the regulus generated by swinging the prismatic joint about the axis of the revolute, see Fig. 2.

The equation satisfied by the group elements satisfying this constraint can be found in much the same way as in the previous section. In this case we need to consider the distance of the point to the axis of the revolute joint, $(p \vee \ell)(p \vee \ell)^{-}$as well as the distance from the point to the plane defined by the common perpendicular between the axes of the revolute and prismatic joints, see Fig. 2. If this plane is labelled $\pi$ then the square of the perpendicular distance from the point $p$ to the plane is given by the expression, $(p \vee \pi)(p \vee \pi)^{-}$. The equation of a general hyperbola is $x^{2} / \alpha^{2}-y^{2} / \beta^{2}=1$, where $\alpha$ and $\beta$ are constants. Hence the group elements $g$, which preserve the incidence of the point with a cylindrical hyperboloid will be,

$$
\frac{1}{\alpha^{2}}\left(g p g^{-} \vee \ell\right)\left(g p g^{-} \vee \ell\right)^{-}-\frac{1}{\beta^{2}}\left(g p g^{-} \vee \pi\right)\left(g p g^{-} \vee \pi\right)^{-}=\left(g g^{-}\right)^{2}
$$

As in the previous section this constraint equation has degree 4 in the components of the group element $g$. 
Suppose that the minimum distance from the axis of the revolute joint $\ell$ to the point $p$ is $l$. This design parameter will be referred to as the link-length of the linkage. Clearly this minimum will occur when the point lies on the plane $\pi$ and hence it is easy to see that $\alpha=l$ in the equation above. By considering a more general point on the hyperboloid, see fig.2(b), the constant $\beta$ can be shown to satisfy $\beta^{2}=l^{2} / \tan ^{2} \phi$, where $\phi$ is the twist angle between the axes revolute joint and the prismatic joint. Hence in terms of the design parameters $l$ and $\phi$ the equation for the constraint variety may be written as,

$$
\cos ^{2} \phi\left(g p g^{-} \vee \ell\right)\left(g p g^{-} \vee \ell\right)^{-}-\sin ^{2} \phi\left(g p g^{-} \vee \pi\right)\left(g p g^{-} \vee \pi\right)^{-}=l^{2}\left(g g^{-}\right)^{2}
$$

As a concrete example consider a linkage with a similar design to the CS linkage studied above. The point $p$ can initially be taken to be at the origin, $p=e_{1} e_{2} e_{3}$ and the axis of the revolute joint can be taken as $\ell=e_{1} e_{2}-l e_{2} e$, that is a line parallel to the $z$-axis but displaced $l$ units in the $x$-direction. The plane $\pi$ can be taken as the $x y$-plane, $\pi=e_{3}$. Notice that the location of the prismatic joint is not important, only its direction determined by the twist angle $\phi$. With these choices many of the computations we need have already been done above, in fact the only new result needed is,

$$
\mathrm{gpg}^{-} \vee \pi=-2\left(a_{0} c_{3}+a_{1} c_{2}-a_{2} c_{1}-a_{3} c_{0}\right) .
$$

Substituting the results into (3) above and rearranging a little gives,

$$
\begin{aligned}
0= & 4 \cos ^{2} \phi\left(a_{0} c_{2}-a_{1} c_{3}-a_{2} c_{0}+a_{3} c_{1}\right)^{2}+ \\
& 4 \cos ^{2} \phi\left(a_{0} c_{1}-a_{1} c_{0}+a_{2} c_{3}-a_{3} c_{2}\right)^{2}- \\
& 4 \sin ^{2} \phi\left(a_{0} c_{3}+a_{1} c_{2}-a_{2} c_{1}-a_{3} c_{0}\right)^{2}- \\
& 4 l \cos ^{2} \phi\left(a_{0} c_{1}-a_{1} c_{0}+a_{2} c_{3}-a_{3} c_{2}\right)\left(a_{0}^{2}+a_{1}^{2}+a_{2}^{2}+a_{3}^{2}\right)- \\
& l^{2} \sin ^{2} \phi\left(a_{0}^{2}+a_{1}^{2}+a_{2}^{2}+a_{3}^{2}\right)^{2} .
\end{aligned}
$$

Notice that the quartic variety in $\mathbb{P}^{7}$ defined by this equation contains the 3-plane $A_{\infty}$ and moreover is singular on this plane.

\section{The RRS Linkage}

Finally here consider the RRS linkage. It is well known that a point attached to a pair of revolute joints will trace out a general torus, see [1]. As in [1] the equation of the surface traced out by the point can be found by considering the radial components and $z$-components of the point $p$ in fig. 3 and then eliminating the second joint angle $\theta_{2}$ to give,

$$
\left(\left(x^{2}+y^{2}+z^{2}\right)-\left(l^{2}+d^{2}+r^{2}\right)\right)^{2}=4 l^{2}\left(r^{2}-\left(\frac{z-d \cos \phi}{\sin \phi}\right)^{2}\right) .
$$




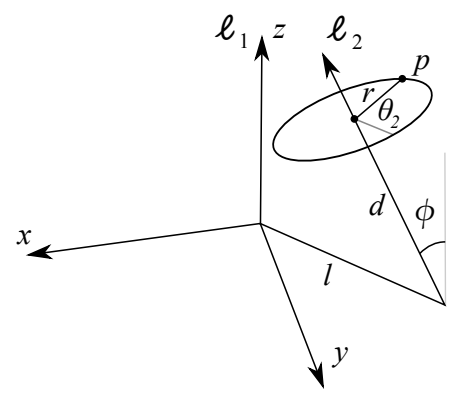

Fig. 3 The RRS linkage.

The design parameters $l, d, r$ and $\phi$ are as shown in fig.3and can be thought of as the link-length, offset, second radius and twist-angle respectively.

To find the equation in $\mathbb{P}^{7}$ satisfied by the group elements which preserve the incidence of the point with the torus we look again at the radial and $z$-components of $p$. The radial component of $p$ is the distance from the first joint axis $\ell_{1}$ to $p$. For a general point the square of this distance will be $\left(\mathrm{gpg}^{-} \vee \ell_{1}\right)\left(\mathrm{gpg}^{-} \vee \ell_{1}\right)^{-}$. For the coordinates of fig. 3 , this expression can be used to replace $x^{2}+y^{2}$. The $z$-component of $p$ is the distance to $p$ from the plane containing the perpendicular common to $\ell_{1}$ and $\ell_{2}$. This distance can be expressed as $\mathrm{gpg}^{-} \vee \pi$ which can be used to replace $z$ in the equation above. Multiplying terms by the factor $g^{-}$to produce a homogeneous equation gives,

$$
\begin{array}{r}
\left(\left(g p g^{-} \vee \ell_{1}\right)\left(g p g^{-} \vee \ell_{1}\right)^{-}+\left(g p g^{-} \vee \pi\right)\left(g p g^{-} \vee \pi\right)^{-}-\left(l^{2}+d^{2}+r^{2}\right)\left(g g^{-}\right)^{2}\right)^{2}= \\
4\left(g g^{-}\right)^{2} l^{2}\left(\left(g g^{-}\right)^{2} r^{2}-\left(\frac{\left(g p g^{-} \vee \pi\right)-d\left(g g^{-}\right) \cos \phi}{\sin \phi}\right)^{2}\right)
\end{array}
$$

Clearly this equation has degree 8 in the components of the group elements $g$.

In reference [1] Fichter and Hunt look for circles in the general torus described above to find all possible mobile $R R S R$ mechanisms. A revolute joint produces a one-parameter family of group elements which lie on a line in the Study quadric. So to find all mobile RRSR mechanisms we could look for lines in the degree 8 hypersurface given by equation (4). Actually, we only need to look for lines of the form $g(\lambda)=1+\lambda \ell$ where $\ell$ is a line - the axis of the final revolute joint. Substituting this form into the equation for the constraint variety will produce a degree 8 equation in the parameter $\lambda$. For the line to lie in the constraint variety the coefficients of each power of $\lambda$ must vanish. Hence we can produce conditions for an RRSR mechanism to be mobile. In principle, this strategy for finding mobile mechanisms can be extended to other linkages, however the analysis of the conditions produced may not be straightforward. 
5 The Segre Variety $\mathbb{P}^{1} \times \mathbb{P}^{1} \times \mathbb{P}^{3}$

The three examples studied above, together with a couple of other examples not considered here, are united by the fact that their constraint varieties are projections of the Segre variety $\mathbb{P}^{1} \times \mathbb{P}^{1} \times \mathbb{P}^{3}$.

To see this first consider the Segre variety $\mathbb{P}^{1} \times \mathbb{P}^{1} \times \mathbb{P}^{3}$. A point, with homogeneous coordinates, $\left(\alpha_{0}: \alpha_{1}\right) \times\left(\beta_{0}: \beta_{2}\right) \times\left(\gamma_{0}: \gamma_{1}: \gamma_{2}: \gamma_{3}\right)$ can be mapped to a point $\left(X_{000}: X_{100}: X_{010}: \cdots: X_{113}\right)$ in $\mathbb{P}^{15}$. This is the Segre map defined by $X_{i j k}=\alpha_{i} \beta_{j} \gamma_{k}$. That is $X_{000}=\alpha_{0} \beta_{0} \gamma_{0}, X_{100}=\alpha_{1} \beta_{0} \gamma_{0}$ and so forth. The image of this map is known as the Segre variety. Using the techniques described in [2] it is possible to show that this variety has degree 20 . It is also easy to see that it lies on several quadric hypersurfaces in $\mathbb{P}^{15}$. There are 12 quadrics of the form $X_{i j k} X_{i l m}-X_{i j m} X_{i l k}=0$ and another 12 of the form $X_{i j k} X_{l j m}-X_{i j m} X_{l j k}=0$. Then there are 6 each of the forms $X_{00 k} X_{11 l}-X_{10 l} X_{01 k}=0, X_{01 k} X_{10 l}-X_{00 l} X_{11 k}=0$ and $X_{00 k} X_{11 l}-X_{01 k} X_{10 l}=0$. Finally there are 4 of the form $X_{00 k} X_{11 k}-X_{10 k} X_{01 k}=0$. This makes 46 linearly independent quadrics.

To see the connection with the constraint varieties discussed above we will look at just one example. Consider the CS dyad from Section 2, in particular the example given at the end of the section. The constraint variety can be parameterised as a product of Clifford algebra elements,

$$
g=\left(\alpha_{0}+\alpha_{1}\left(e_{1} e_{2}-r e_{2} e\right)\right)\left(\beta_{0}+\beta_{1} e_{3} e\right)\left(\gamma_{0}+\gamma_{1} e_{2} e_{3}+\gamma_{2} e_{3} e_{1}+\gamma_{3} e_{1} e_{2}\right) .
$$

Multiplying out the above product gives an element of the form given in (2) with components,

$$
\begin{array}{ll}
a_{0}=\alpha_{0} \beta_{0} \gamma_{0}-\alpha_{1} \beta_{0} \gamma_{3}, & c_{0}=-\alpha_{0} \beta_{1} \gamma_{3}-\alpha_{1} \beta_{1} \gamma_{0}+r \alpha_{1} \beta_{0} \gamma_{2}, \\
a_{1}=\alpha_{0} \beta_{0} \gamma_{1}-\alpha_{1} \beta_{0} \gamma_{2}, & c_{1}=-\alpha_{0} \beta_{1} \gamma_{2}-\alpha_{1} \beta_{1} \gamma_{1}-r \alpha_{1} \beta_{0} \gamma_{3}, \\
a_{2}=\alpha_{0} \beta_{0} \gamma_{2}+\alpha_{1} \beta_{0} \gamma_{1}, & c_{2}=\alpha_{0} \beta_{1} \gamma_{1}-\alpha_{1} \beta_{1} \gamma_{2}-r \alpha_{1} \beta_{0} \gamma_{0}, \\
a_{3}=\alpha_{0} \beta_{0} \gamma_{3}+\alpha_{1} \beta_{0} \gamma_{0}, & c_{3}=\alpha_{0} \beta_{1} \gamma_{0}-\alpha_{1} \beta_{1} \gamma_{3}+r \alpha_{1} \beta_{0} \gamma_{1} .
\end{array}
$$

Now suppose we choose new coordinates for $\mathbb{P}^{15}$, label these new coordinates $\bar{a}_{0}, \ldots, \bar{a}_{3}, \bar{b}_{0}, \ldots, \bar{b}_{3}, \bar{c}_{0}, \ldots, \bar{c}_{3}$ and set,

$$
\bar{a}_{0}=X_{000}-X_{103}, \quad \bar{c}_{0}=-X_{013}-X_{110}+r X_{102},
$$

and so forth. The coordinates $\bar{b}_{i}$ can be chosen so that the coordinate transformation is non-singular. Now it is clear that mapping $\bar{a}_{i} \mapsto a_{i}$ and $\bar{c}_{i} \mapsto c_{i}$ maps the Segre variety to the constraint variety for the CS linkage. Geometrically this is a linear projection from $\mathbb{P}^{15}$ to $\mathbb{P}^{7}$, the centre of the projection is given by the 7-plane which is the intersection of the eight hyperplanes $\bar{a}_{i}=0, \bar{c}_{i}=0$. It is not difficult to see that similar constructions can be given for the other examples discussed above.

Note that is should be possible to compute the degree of the constraint variety from the degree of the Segre variety and a knowledge of how the Segre variety meets the centre of the projection, see [2]. 


\section{Conclusions}

In the space available it has only been possible to outline briefly the geometry of these constraint varieties and hint at some possible applications for this approach.

This work should be viewed as part of a general programme to investigate geometric constraint varieties. These are the possible rigid displacements allowed by some geometric problem. The rigid displacements allowed by simple linkages are of most relevance to Kinematics and Robotics. It is known that a revolute joint produces a line in the Study quadric, a general RR dyad generates the intersection of the Study quadric with a 3-plane. An RRR linkage gives a $\mathbb{P}^{1} \times \mathbb{P}^{1} \times \mathbb{P}^{1}$ Segre variety, [3]. Some quadratic constraint varieties were studied in [7].

The ubiquity of Segre manifolds is now clear and connected to the parameterisation of these varieties as products of subgroups. The constraint variety of a general $R R$ dyad could be thought of as the Segre variety $\mathbb{P}^{1} \times \mathbb{P}^{1}$. Moreover, it can be predicted with some confidence that the constraint variety formed by the displacements allowed by a general RRRR linkage will be the projection of a $\mathbb{P}^{1} \times \mathbb{P}^{1} \times \mathbb{P}^{1} \times \mathbb{P}^{1}$ Segre variety.

By intersecting several of these constraint varieties we can study the properties of mechanisms formed by joining the corresponding linkages in parallel. Except in the case of point-plane constraints, this problem has not been investigated to any great extent.

McCarthy and co-workers use equations such as those found above to design mechanisms, see [4]. It would be useful to formalize this problem by embedding the space of all possible linkages of some type, in an algebraic variety. For example, the CS dyads could be thought of as (an open set in) $Q_{K} \times \mathbb{P}^{3}$, the product of the Klein quadric of lines in $\mathbb{P}^{3}$ with the space of points. This turns design synthesis into a problem in Algebraic geometry. Unfortunately these problems are still highly non-trivial.

\section{References}

1. E.F Fichter, E.F. and Hunt, K.H., The fecund torus, its bitangent-circles and derived linkages, Mechanism and Machine Theory, 10(2-3):167-176, (1975). doi:10.1016/0094-114X(75)900178

2. Harris, J., Algebraic Geometry a first course, Springer Verlag, New York, (1992).

3. Husty, M.L., Pfurner, M. and Schröcker, H-P. A new and efficient algorithm for the inverse kinematics of a general serial 6R manipulator, Mechanism and Machine Theory, 42(1):66-81, (2007). doi:10.1016/j.mechmachtheory.2006.02.001

4. McCarthy, J.M. and Soh, G.S., Geometric Design of Linkages, 2nd ed. Springer Verlag, New York, (2011).

5. Selig, J.M., Geometric Fundamentals of Robotics, Springer Verlag, New York, (2005).

6. Selig, J.M., On the geometry of point-plane constraints on rigid-body displacements, Acta Applicandae Mathematicae, 116(2):133-155, (2011). doi:10.1007/s10440-011-9634-6

7. Selig, J.M., Quadratic constraints on rigid-body displacements, ASME Journal of Mechanisms Robotics, 2(4):041009 (7 pages), (2010). doi: 10.1115/1.4002344 